\title{
Risk preferences and risk perception affect the acceptance of digital contact tracing
}

Rebecca Albrecht ${ }^{1,3 凶}$, Jana B. Jarecki ${ }^{1,3}$, Dominik S. Meier ${ }^{1,2} \&$ Jörg Rieskamp ${ }^{1}$

Digital contact-tracing applications (DCTAs) can help control the spread of epidemics, such as the coronavirus disease 2019 pandemic. But people in Western societies fail to install DCTAs. Understanding the low use rate is key for policy makers who support DCTAs as a way to avoid harsh nationwide lockdowns. In a preregistered study in a representative German-speaking Swiss sample $(N=757)$, the roles of individual risk perceptions, risk preferences, social preferences, and social values in the acceptance of and compliance with DCTA were compared. The results show a high compliance with the measures recommended by DCTAs but a comparatively low acceptance of DCTAs. Risk preferences and perceptions, but not social preferences, influenced accepting DCTAs; a high health-risk perception and a low data-security-risk perception increased acceptance. Additionally, support of political measures, technical abilities, and understanding the DCTA functionality had large effects on accepting DCTAs. Therefore, we recommend highlighting personal health risks and clearly explaining DCTAs, focusing on data security, to enhance DCTA acceptance.

\footnotetext{
${ }^{1}$ Department of Psychology, University of Basel, Basel, Switzerland. ${ }^{2}$ Center for Philanthropy Studies, University of Basel, Basel, Switzerland. ${ }^{3}$ These authors

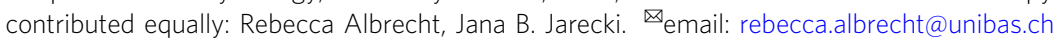




\section{Introduction}

powerful intervention that can help mitigate the spread of a pandemic such as the coronavirus disease (COVID-19) pandemic is digital contact tracing through mobile applications (hereafter, DCTAs). DCTAs use a mobile device's Bluetooth connection to trace the network of close contacts among people by anonymously recording the locations of mobile devices that are running the DCTA in the vicinity of a DCTA user. The DCTA's purpose is to alert people in this network who have had close contact with an infected person. As long as vaccines and medical treatments for a disease are under development or not widely available, non-pharmaceutical, behavioural interventions are the main means to contain the spread of a pandemic. In the case of COVID-19, such interventions include DCTAs, isolation of symptomatic individuals, quarantine of people who have come in contact with an infected person, the closure of schools and universities, and full lockdowns. Some of these measures have dramatic negative economic and psychological costs for society and individuals (Alvarez et al., 2020; Brooks, 2020). Less extreme interventions can therefore be beneficial for maintaining ordinary daily life. Moreover, findings have shown that DCTAs have the potential to contain the spread of a pandemic (Ferretti, 2020; Salathe, 2020) and to forecast future pandemic hot spots (Menni, 2020). Beyond the 2020 COVID-19 outbreak, DCTAs provide a powerful yet readily available tool to mitigate the spread of future pandemics at an early stage. But importantly, for DCTAs to work effectively, a large proportion of the population must use them (Xia and Lee, 2020). Yet people in Western countries remain sceptical about DCTAs for reasons including privacy concerns (Akinbi et al., 2020; Blasimme and Vayena, 2020; Jansen-Kosterink, 2020; Park et al., 2020). In Switzerland, for instance, only around $27 \%$ of the population was actively using the Swiss federal DCTA as of March 2021 (Federal Statistical Office, 2021) and only $32 \%$ of the German population had the respective DCTA installed (Robert Koch Institut, 2021). Therefore research is needed to understand the factors affecting DCTAs' acceptance and to inform policy makers who hope to foster their use (Blasimme and Vayena, 2020). Here, use of DCTAs refers to installing and activating a DCTA.

In a recent commentary on good governance of digital contact tracing (Blasimme and Vayena, 2020), policy makers have been advised to employ a reflexive adaptation strategy; this strategy involves gathering data to evaluate when, how, and why people actually use DCTAs. Such data include how people respond to DCTA alerts and perceive the associated risks. Therefore, in the present research, we present data on the psychological factors that shape people's willingness to accept DCTAs and on their compliance with the recommendations of the DCTA.

Individual and social factors affecting the acceptance of behavioural and non-pharmaceutical interventions. From an individual perspective, the decision whether to engage in risky health behaviours during a virus pandemic such as meeting with a large group inside should depend on one's risk perception and risk preferences (Van der Pligt, 1996; Weber and Milliman, 1997) and one's general risk knowledge (Weinstein and Lyon, 1999). Regarding the COVID-19 pandemic, risk mitigation behaviours have been linked to knowledge about SARS-CoV-2, the virus causing the disease (Kwok, 2020; Zhong, 2020), and the perception of associated risks (Abdelrahman, 2020; Betsch et al., 2020; Dryhurst, 2020; Glockner, 2020; Plohl and Musil, 2021; Wise, 2020; Xie, 2020). Individual differences in risk preferences (Frey, 2017) may explain why people with similar risk perception and understanding respond differently, with risk-averse people being less likely to take risks than risk-tolerant people. The pandemic also represents an economic risk to the individual, because behavioural interventions in general can have severe negative economic consequences (Alvarez et al., 2020). The use of DCTAs could also be associated with data-security risks for the individual; in various countries the development of DCTAs has been accompanied by a debate about the data-security threat to individuals (Ienca and Vayena, 2020). Such data-security concerns involved fears of surveillance, data privacy violations, and continuous location data collection. This debate has led to major changes in the development of DCTAs (Beskorovajnov et al., 2020) to reduce the data-security concerns: Rather than collecting location data, the Swiss DCTA is designed to track contacts by a phone-to-phone exchange of a random anonymous code that the application generates on demand when a pair of mobile devices with DCTAs find themselves in close proximity. The pair of devices exchange the respective codes via Bluetooth and the codes are stored locally on the mobile devices. Given these different potential risk factors, from the perspective of the individual, the acceptance of specific non-pharmaceutical interventions might depend on people's risk preferences and the perceptions of the risks they perceive the different interventions to involve.

Besides the individual perspective on DCTAs, the use of DCTAs can also be viewed as a means to manage the capacity of the health system. This implies that there is a societal perspective on the acceptance of DCTAs. A crisis such as the COVID-19 pandemic represents a threat to the health system that can be mitigated through DCTA use. From a societal perspective, DCTA use may help prevent a situation in which an exponential growth in the number of infected cases causes the number of people requiring treatment in intensive care units to exceed capacity. From this societal perspective, the health system, and specifically intensive care unit capacity, can be viewed as a common-pool resource (Ostrom, 1990) that has to be managed sustainably to prevent overuse. DCTAs present one way to manage this resource, and acceptance of DCTAs can be viewed as an act of cooperation and investment in the common pool. Past work has shown that the over-harvesting of common pools is especially prevalent in social groups that contain a substantial number of 'free riders', that is, people who take benefits without paying any costs (Camerer, 2003). Whether a common-pool resource can be managed sustainably depends on people's social preferences (Falk and Fischbacher, 2006; Fehr and Schmidt, 1999). Results of research into COVID-19 (Campos-Mercade, 2021) have demonstrated that prosocial preferences affect the engagement in preventive behaviours such as maintaining physical distance and self-isolation (Dryhurst, 2020; Zettler, 2021). Furthermore, Bavel et al. (2020) have distinguished different societal collectives in the pandemic, such as families, communities, nations, and international regions, and people might give different priorities to these collectives (Chen and $\mathrm{Li}, 2009$ ) when following nonpharmaceutical interventions. It is known that investment in common resources tends to decrease in more heterogeneous groups, with trust as a psychological mediator (e.g., van Klingeren, 2020). Taken together, these results suggest that from this societal perspective on the acceptance of DCTAs as an act of cooperation in a common-pool dilemma, individuals' social preferences, their identification with communities, and their trust in the governmental management of the COVID-19 crisis should affect the acceptance of and compliance with DCTAs. Figure 1 illustrates the effects of these social and individual factors.

Critically, the societal and the individual perspectives on pandemics such as the COVID-19 pandemic need not be aligned. Particularly, younger people may perceive COVID-19 as less risky for their own health and consequently might not follow severe non-pharmaceutical interventions to fight the pandemic that are 


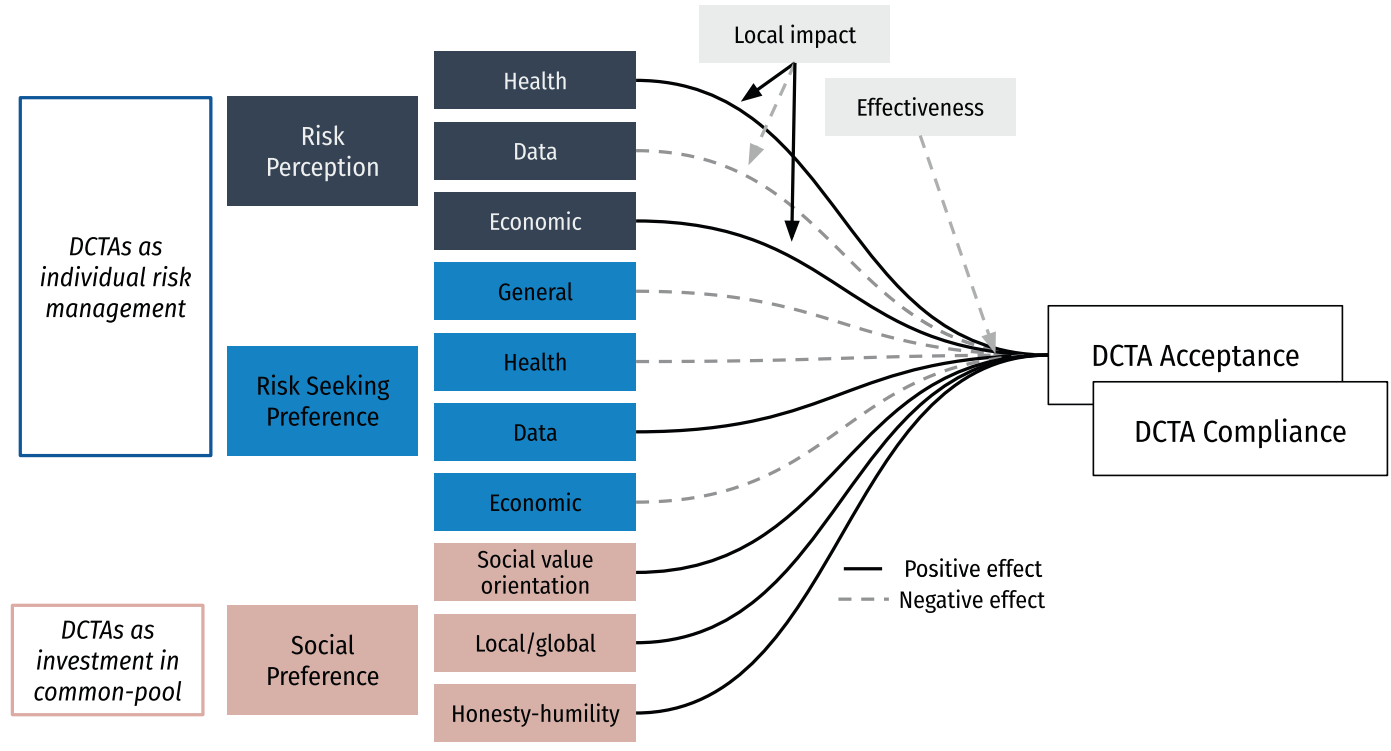

Fig. 1 Preregistered hypotheses. Predictions based on the individual and the societal perspective regarding the role of risk preferences, risk perceptions, and social preferences on digital contact-tracing application (DCTA) use.

necessary from the societal perspective. A person's decision to reject non-pharmaceutical interventions, which can be reasonable from the individual perspective regarding the risks of COVID-19 to the self, could foster the transmission of the virus and therefore hamper public health.

Aim of this research. The present study examined the predictive power of the psychological factors leading people to accept DCTAs as a measure to manage the COVID-19 pandemic and to comply with a DCTA's suggestions regarding safety measures (e.g., staying home after close contact with an infected person). The novel contribution of our work consists in its theory-driven comparison between social and individual factors that affect DCTA acceptance and compliance. Acceptance refers to the willingness to use and recommend DCTAs and compliance refers to following the DCTA's recommendations if the DCTA alerts about a prior contact with an infected person. Specifically, we focus on the individual factors risk perception and risk preference within the relevant domains of health, economy, and data security and compare the individual factors to different social factors such as social value orientation and prosociality. The acceptance of and compliance with DCTAs can also be affected by other factors, such as the comprehension of DCTAs and the locality of the perceived threat, among others. The study design and hypotheses have been preregistered at https://osf.io/b3ud5.

\section{Methods}

The empirical investigation tested the psychological factors that affect (i) the acceptance of DCTAs as a means to manage the COVID-19 pandemic and (ii) the compliance with the recommended behaviour from a DCTA, such as self-isolation. In particular, we focused on the role of the psychological constructs social preferences (how much do I care about others?), risk perception (how high does a risk seem to me?), and risk preferences (how much risk do I tolerate?). The methods (design and hypotheses, sample size, and analysis strategy) have been preregistered (see https://osf.io/b3ud5); they are available online together with the data and the materials (https://osf.io/u6ngf/).

Preregistered hypotheses. A summary of the hypotheses is presented in Fig. 1. Regarding the societal perspective, we hypothesised that the higher the general other-regarding social preferences are, the higher the acceptance of and compliance with DCTAs will be. The higher a person scores on the personality trait honesty-humility, the higher their acceptance of and compliance with DCTAs will be. The higher the social preference for the local versus the global community is, the higher the acceptance and compliance with DCTAs. Regarding the individual perspective, we hypothesised domain-specific effects of risk preferences such that high health-risk perception will increase the acceptance of and compliance with DCTAs, but high datasecurity-risk perception will decrease the acceptance of and compliance with DCTAs. High economic-risk perception should increase the acceptance of and compliance with DCTAs, because widespread use of DCTAs can help communities avoid hard measures such as lockdowns, which lead to severe economic downturns (Kochanczyk and Lipniacki, 2021). Regarding risk preferences, we hypothesised general and domain-specific effects, namely, that a high general risk tolerance will decrease the acceptance of and compliance with DCTAs, and that high healthrisk tolerance will decrease the acceptance of and compliance with DCTAs, but high data-security-risk tolerance will increase the acceptance of and compliance with DCTAs. High economicrisk tolerance was hypothesised to decrease the acceptance of and compliance with DCTAs.

Participants. The data came from a large nationally representative sample of the Swiss German-speaking population, recruited through a panel provider (LINK Institute, Lucerne, Switzerland) that drew an age- and gender-representative sample from adults residing in the German-speaking part of Switzerland. Participants responded to an online questionnaire assessing the acceptance of and compliance with the Swiss federal contact-tracing application ('SwissCovid'); the data were collected in June 2020 (after the first wave of SARS-CoV-2 infections in Switzerland and one week after the Swiss DCTA became available). The design was approved by the ethics committee of the Faculty of Psychology at the University of Basel. In total, 848 participants completed the questionnaire; 91 had to be excluded (as preregistered) because of low data quality, ${ }^{1}$ leaving a final sample of $N=757$ (388 men, 366 women, 3 did not report gender; $51.3 \%, 48.3 \%$, and $0.4 \%$, respectively); the mean age was 45 years $(M d n=44$ years, 
$S D=16$, range $18-79$ years), and $65 \%$ had at least a high school diploma (please see Supplementary Table S1 for further demographic information).

Participants received a remuneration of 3.00 Swiss francs (ca. 3.25 U.S. dollar at the time of the study) for completing the survey, and $10 \%$ of participants were randomly selected to receive a bonus payment that depended on their response to the social value game-theoretic question (see below) in which participants had to divide 1-1.7 Swiss francs between themselves and an unknown person. This served to incentivise the social preference measure. $^{2}$

Materials. The survey was implemented using the online survey software Qualtrics (Qualtrics, Provo, UT). The dependent variables-acceptance of and compliance with the Swiss DCTA (SwissCovid) - were assessed by four items each: The acceptance scale measured the inclination to use and to recommend and the perceived effectiveness and data security of the DCTA on a 5 -point Likert-type scale $(1=$ disagree completely, $3=$ neutral, 5 = agree completely). The compliance scale included the willingness to self-isolate, report a positive diagnosis, call the national hotline, and get tested following a critical contact alert by the DCTA. The wording can be found in the Supplementary material. The scale responses were averaged by participant into their acceptance and compliance score. Before reporting their acceptance and compliance, participants read a brief one-paragraph vignette that explained contact tracing and that the SwissCovid application detected close contacts. The information and wording presented in the vignette was taken from the Federal Office of Public Health of the Swiss Confederation (Federal Office of Public Health, 2020a).

Social preferences were measured using the following standardised measures of trait and state social preferences: The social value orientation was measured by Murphy et al.'s (2011) six mini dictator games, in which participants divide money between themselves and another person. An angle is computed from the choices in these games (see Murphy et al., 2011, for details), with larger angles indicating higher prosociality. Other-regarding personality facets were measured by the honesty-humility subscale of the brief HEXACO personality inventory (de Vries, 2013); participants' identification with the world versus the local community was assessed by the identification with all humanity scale (IWAH; McFarland et al., 2012). The HEXACO and the IWAH subscales were each averaged and $z$ standardised by participant.

Risk preferences were measured by items from the largest German household socio-economic panel (SOEP; see Frey, 2017, Infratest Sozialforschung, 2009) on a 10-point Likert-type scale $(0=$ not willing to take risks at all to $10=$ very willing to take risks). One additional item was added to measure data-security risks, since this domain is not part of the German SOEP survey. The SOEP responses were $z$ standardised by participant.

Perception of the health risks of COVID-19 was assessed by three items (following Weinstein and Lyon, 1999): the number of people out of 100,000 people in Switzerland (i) who have been infected during the last 7 days and (ii) who will get infected during the next 7 days, and (iii) the number of people out of 100 infected people in Switzerland who will develop a severe course of disease. The numeric responses were $z$ standardised across participants.

The main covariates of interest included demographic variables such as gender, age, income, and wealth as well as the comprehension of DCTAs, the willingness to adopt new technologies (technology affinity, measured on a four-item scale) and support for political measures against the 2020 COVID pandemic in general (policy support, measured on a four-item scale). Comprehension of the SwissCovid application was measured on a four-item scale asking, for instance, if the data were stored anonymously (the wording can be found in the supplementary material). The multi-item scales were averaged and $z$ standardised by participant for the analysis.

Statistical analyses. Because the questionnaire allowed for nonresponses regarding income and wealth (see Table S1), missing values were imputed by the median sample income and wealth (respectively). This imputation was not preregistered. Following our preregistered analysis strategy, we tested the hypotheses using Bayesian linear regressions by modeling the acceptance of and compliance with DCTAs as a function of the theoretically relevant predictors (risk perception variables, risk preference variables, social preference variables) and selected covariates, where the covariates were selected to yield the most parsimonious predictive model (see covariate selection). All variables were $z$ standardised before the analyses.

Covariate selection. The covariates in the regression model were selected using a Bayesian projective predictive model selection method (Vehtari and Ojanen, 2012). This model selection method has been shown to outperform other methods in selecting the variables that balance model sparsity and predictive accuracy (Pavone et al., 2020; Piironen et al., 2020). This variable selection method constructs a reference model (we used the full model including all possible covariates) and searches for a reduced model with minimal loss of performance compared to the reference model. The simpler model is constructed by projecting the model parameters from the full model, because exhausting all possible combinations of predictor variables is infeasible. The predictive projection was used to select the covariates to be included in the regression; the variables related to risk perceptions, risk preferences, and social preferences were defined as variables to be retained as predictors in the model for as long as possible (using a Lasso-type L1-penalisation; details of this method are given in Piironen et al., 2020).

\section{Results}

In addition to presenting the first rigorous comparison of individual and societal psychological factors relevant to DCTAs, we go beyond previous descriptions of subjective risk perceptions related to the COVID-19 pandemic (e.g., Karlsson, 2021; Plohl and Musil, 2021) and present results that can provide a detailed account of the factual knowledge about the risks associated with COVID-19 in Switzerland as of June 2020, including incidence rates, symptoms, and risk factors (Weinstein and Lyon, 1999). Our results complement previous studies that have looked at the acceptability of digital contact-tracing technologies in different countries (Garrett, 2021a, b; Lewandowsky, 2021) and add to this literature because our results refer not only to individual but also to societal psychological factors (i.e., social preferences).

The perception of COVID-19. The majority of the sample viewed the COVID-19 pandemic as a problem for the entire world (92\%), but less than half (42\%) regarded it as a problem for the immediate vicinity or themselves, and many viewed it as no problem in the vicinity (36\%; the remaining $22 \%$ were undecided). At the time of the survey (June 2020), 1.7\% of respondents reported a positive diagnosis of COVID-19, and $11 \%$ reported that they or a close contact had received a positive diagnosis in the past. Among the people who reported neither a current infection nor past contact with an infected person, 26\% reported at least one COVID-19-related symptom (fever, sore throat, dry 
A1

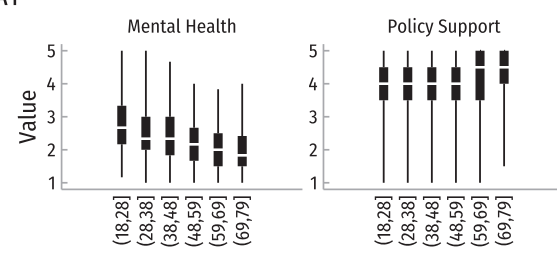

B1
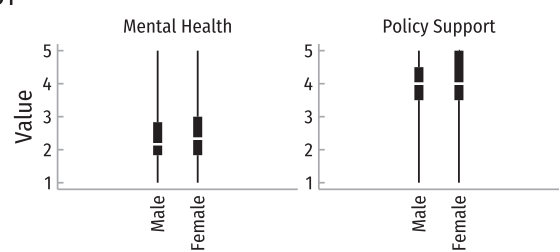

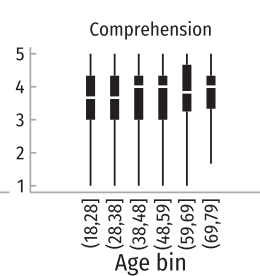

Age bin
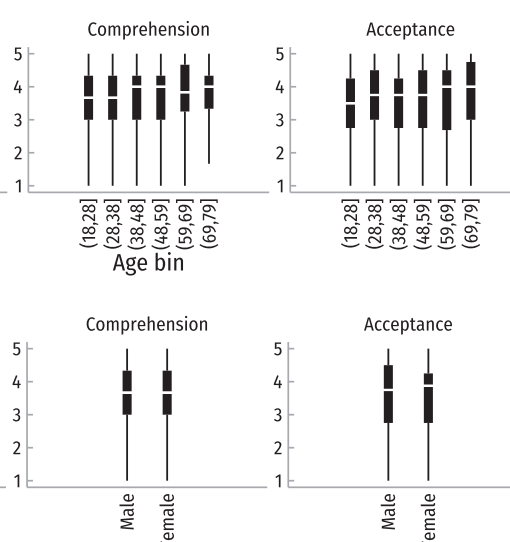

Comprehension

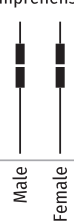

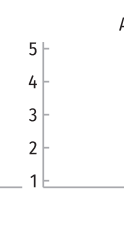

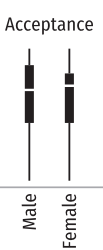

A2

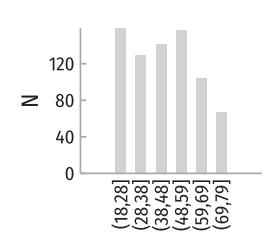

B2

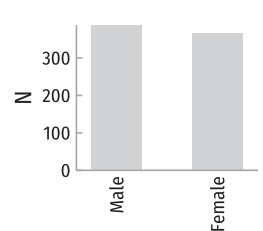

Fig. 2 Descriptive information. Mental health in the last month before the study, support for pandemic-mitigating political measures, comprehension of the functionality of digital contact-tracing applications (DCTAs), acceptance of DCTAs, and compliance with DCTAs. (A1) Age differences. (B1) Gender differences. (A2, B2) Frequency distribution of age groups and gender in the sample ( $n=3$ gender non-responses were excluded in B1 and B2).

cough, shortness of breath, muscle pain, or sudden loss of sense of smell or taste). The majority of respondents (93\%) indicated that they were working from home either fully or part-time, and $15 \%$ reported a reduction in their income due to the COVID-19 pandemic. Figure 2 shows age and gender differences regarding the respondents' mental well-being and their support of the ongoing political measures against COVID-19. On a scale of 1-5, women supported the political measures slightly more $(M=4.09$, $S D=0.84)$ than men $(M=3.86, S D=1.00)$, and the older cohorts (above 69 years; $M=4.30, S D=0.87$ ) slightly more than the youngest cohort (up to 28 years; $M=3.86, S D=0.90$ ).

Risk knowledge regarding COVID-19. Around half of the respondents $(M=49 \%$ correct) knew the absolute number of COVID-19-related deaths, which equalled 1750 at the time of the survey, and one third (38\%) knew the absolute number of infections, which equalled 32,500 at the time of the survey. They were well-informed about the pre-existing conditions associated with a severe illness caused by COVID-19: Nearly everybody (97\%) correctly identified chronic respiratory diseases and $68 \%$ identified cancer as a risk factor, which was the least-identified risk factor; kidney disease was the most frequently misidentified risk factor (29\%). The risk factors were defined according to the Swiss FOPH (Federal Office of Public Health, 2020b). A third of the participants $(31 \%)$ correctly stated the past-7-day incidence rate, but many participants (44\%) overestimated it. The past-7day incidence is the cumulative number of new infections relative to 100,000 inhabitants in the 7 days prior to the survey and was measured in a natural frequency format as $x$ of 100,000 , because frequencies have been shown to facilitate the understanding of risks compared to, for instance, probability formats (e.g., Hoffrage and Gigerenzer, 1998; Visschers, 2009). A quarter (27\%) correctly predicted the future-7-day incidence rate, which was overestimated by half the sample (51\%). Half of the participants (50\%) believed that the incidence rate would remain unchanged from the past to the next 7 days. Only a minority reported the highest possible incidence rate of 45 per 100,000 inhabitants (12\% past-7day, $12 \%$ future-7-day incidence).

Acceptance of and compliance with DCTAs. On a scale of 1-5, the acceptance of digital contact tracing in the form of the Swiss DCTA averaged 3.75 ( $S D=1.1$, computed based on four items). The mean compliance score of 4.34 (range $1-5, S D=0.82$ ) shows that participants were highly willing to comply with the DCTA recommendation if they were to use the contact-tracing application. The acceptance of DCTAs was lowest for 18-28-yearolds $(M=3.41, S D=1.05)$ and highest for age 69 years and older $(M=3.83, S D=0.93$, Fig. 2). Slightly more than half $(58 \%)$ of the sample agreed that the Swiss DCTA was technically well designed, but most respondents (89\%) did not believe that a sufficient number of people would actually use it. Figure 2 shows age and gender differences regarding respondents' acceptance of, compliance with, and comprehension of the functionality of DCTAs.

Psychological factors that impact the acceptance of and compliance with DCTAs. The effects on the acceptance of DCTAs were estimated with Bayesian regression models using standardised variable scales (effect in units of standard deviations). The resulting regression coefficients (shown in Table 1) seem to indicate that the risk perception variables and risk preference variables had an overall larger effect than the social preference variables (risk perception $\beta$ s from -0.08 to 0.06 , risk preference $\beta$ s from -0.06 to 0.11 , and social preference $\beta$ s from -0.01 to $0.04)$. Therefore we tested if excluding the social variables improved the model using a model comparison, which revealed that exclusion of the social preference variables greatly improved the model fit [Bayes factor $(\mathrm{BF})=20,271,851$ for a model without the social preference variables compared to the full model; the former also outperformed a model excluding risk perceptions, $\mathrm{BF}=2236$, and one excluding risk preferences, $\mathrm{BF}=12]$. Also, we found no evidence of moderation effects: The effects on DCTA acceptance were not moderated by the perceived effectiveness of contact tracing, defined as the mean belief in the technical functioning and sufficient adoption (model comparison, $\mathrm{BF}=7.94$ for a model without effectiveness as moderator ${ }^{3}$ ). Nor were the effects on DCTA acceptance moderated by the perceived threat level of COVID-19 in Switzerland ( $\mathrm{BF}=4.67$ for a model without local threat as moderator). Therefore, the results do not include moderator variables (Table 1).

We can conclude that the social preference variables did not have a substantial effect on the acceptance of DCTAs compared to people's risk perceptions and risk preferences and therefore, in the following we present the results based on a Bayesian regression model without the social preference variables.

Figure 3 shows the median standardised Bayesian regression coefficients $(\beta \mathrm{s})$, that is, the effect that a change of a predictor by one standard deviation has on one standard deviation of the acceptance of DCTAs. The acceptance of DCTAs was strongly associated with a better understanding of the mode of operation 
Table 1 Results of the Bayesian regression: effects on the acceptance of and compliance with digital contact-tracing applications.

\begin{tabular}{|c|c|c|c|c|c|c|c|}
\hline Group & Predictor & Mdn & \multicolumn{2}{|l|}{$\mathbf{C l}$} & Mdn & \multicolumn{2}{|l|}{$\mathbf{C l}$} \\
\hline \multirow[t]{3}{*}{ Risk perceptions } & Health & 0.06 & 0.02 & 0.10 & -0.01 & -0.07 & 0.04 \\
\hline & Data security & -0.05 & -0.10 & -0.01 & -0.01 & -0.07 & 0.04 \\
\hline & Economic & -0.08 & -0.13 & -0.04 & -0.03 & -0.08 & 0.03 \\
\hline \multirow{2}{*}{ Risk preferences } & Data security & 0.11 & 0.06 & 0.15 & 0.00 & -0.05 & 0.05 \\
\hline & Economic & 0.05 & 0.00 & 0.10 & - & - & - \\
\hline \multirow[t]{3}{*}{ Social preferences } & Honesty-humility & 0.04 & 0.00 & 0.08 & 0.06 & 0.01 & 0.11 \\
\hline & Social value orientation & -0.01 & -0.05 & 0.03 & -0.01 & -0.06 & 0.04 \\
\hline & $\begin{array}{l}\text { Identification with world over } \\
\text { community }\end{array}$ & -0.01 & -0.05 & 0.04 & - & - & - \\
\hline
\end{tabular}

Predictors of the Acceptance of Contact Tracing

A Major Predictors

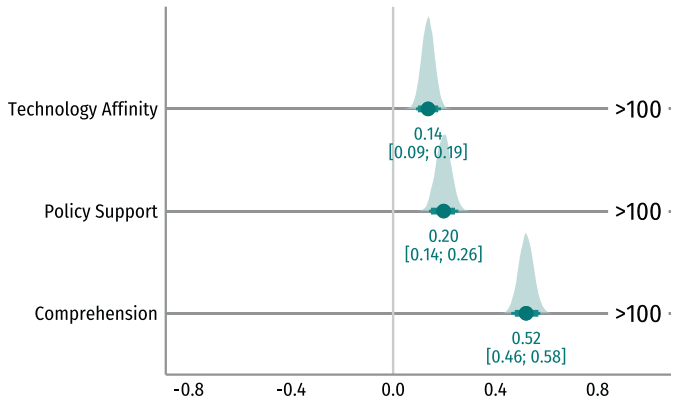

c

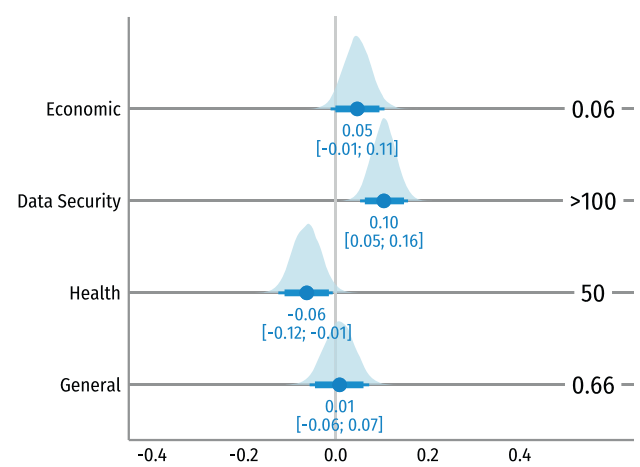

B

Risk Perceptions

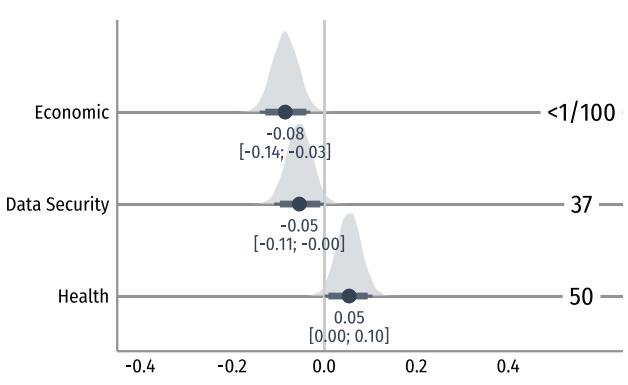

Health-risk Effects
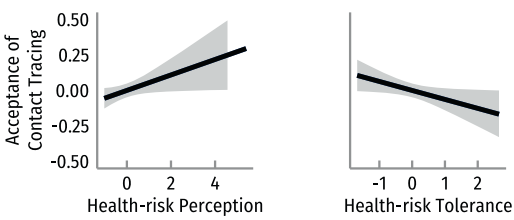

Data-risk Effects
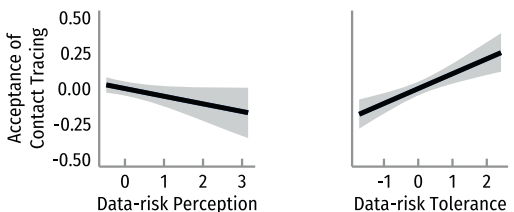

Fig. 3 Factors that influence the acceptance of digital contact-tracing applications. A-C Effects on acceptance of contact tracing (the posterior estimates of regression coefficients). Points $=$ median posterior estimate, interval $=95 \%$ highest density intervals, bold numbers in the lines $=$ Bayes factors in favor of the hypotheses. D Fitted effects of increasing health-risk perception compared to increasing health-risk tolerance and increasing data-security-risk perception compared to data-security-risk tolerance (labelled as data-risk).

of a DCTA $(\beta=0.52)$ and with higher support for general political measures against COVID-19 $(\beta=0.20)$, as shown in Fig. 3A [for the predictor comprehension of DCTAs, the median $\beta=0.52,95 \%$ highest density interval (HDI) $0.46-0.60$, evidence in favor of a positive effect $\mathrm{BF}_{(+)}>100$ based on a normal prior with $M=0$ and $S D=10$; policy support $\beta=0.20$, 95\% HDI $\left.0.14-0.26, \mathrm{BF}_{(+)}>100\right]$.

In terms of psychological factors, accepting DCTAs was positively related to perceiving COVID-19 as a severe health risk $(\beta=0.05)$ and negatively related to perceiving DCTAs as a 
data-security risk $(\beta=-0.05)$, which was in line with the preregistered hypothesis (see the "Methods" section); contrary to the hypothesis, DCTA acceptance was negatively correlated with perceiving COVID-19 as an economic risk $(\beta=-0.08)$ (see Fig. $3 \mathrm{~B}$ ) (health-risk perception, $\beta=0.05$, HDI $0.00-0.10, \mathrm{BF}_{(+)}=50$; data-security-risk perception, $\beta=-0.05,95 \% \mathrm{HDI}-0.11$ to 0.00 , $\mathrm{BF}_{(-)}=37$; economic-risk perception $\beta=-0.08$, 95\% HDI -0.14 to $\left.-0.03, \mathrm{BF}_{(+)}<1 / 100\right)$. The negative association of DCTA acceptance with data-security concerns is in line with previous results (Garrett, 2021a, b; Jansen-Kosterink, 2020; Lewandowsky, 2021; Zhang et al., 2020). The positive association between healthrisk perception and DCTA acceptance is also consistent with previous research (Garrett, 2021b; Lewandowsky, 2021).

Besides the association with risk perceptions, the acceptance of DCTAs was also related to risk preferences: The acceptance of DCTAs increased with more aversion to health risks $(\beta=-0.06)$ and more tolerance regarding data-security risks $(\beta=0.10)$. Neither people's general risk preferences nor their economic risk preferences showed a robust association with their acceptance of DCTAs; see Fig. 3C (health-risk preferences $\beta=-0.06$, HDI -0.12 to -0.01 , $\mathrm{BF}_{(-)}=50$; data-security-risk preferences, $\beta=-0.10$, 95\% HDI -0.05 to $0.16, \mathrm{BF}_{(+)}>100$; economic-risk preferences $\beta=0.05,95 \%$ $\mathrm{HDI}-0.01$ to $0.11, \mathrm{BF}_{(-)}=0.06$; general risk preferences $\beta=0.01$, $95 \% \mathrm{HDI}-0.06$ to $\left.0.07, \mathrm{BF}_{(+)}=0.66\right)$.

Further results. We further examined the variables related to support for the political measures in Switzerland and factors influencing the comprehension of DCTAs. People's support for COVID19-mitigating political measures in Switzerland increased with lower mental health $(\beta=-0.16)$ and lower risk perception regarding datasecurity risks, economic risks, and general risks $(\beta=-0.14,-0.11$, and -0.12 , respectively), and higher DCTA comprehension $(\beta=0.38)$, according to an exploratory Bayesian regression (see Supplemental Table S2). Better comprehension of DCTAs, which was a main variable linked to their acceptance, was associated with high policy support, high data-security-risk tolerance, and high interest in new technologies $(\beta=0.40,0.11,0.10$, respectively), and DCTA comprehension decreased for people who were not working from home $(\beta=-0.18)$ and with the perception of DCTAs as a data-security risk $(\beta=-0.14$; see Supplemental Table S3).

\section{Discussion}

The present study examined the psychological factors that impact the acceptance of and compliance with DCTAs as a measure against the COVID-19 pandemic. DCTAs constitute an easy to use yet efficient non-pharmaceutical means to mitigate the spread of a pandemic (Ferretti, 2020; Menni, 2020; Salathe, 2020), but their success depends on widespread use and acceptance of DCTAs in the population. Using a German-speaking Swiss representative survey conducted after DCTAs were made available in Switzerland, we compared the role of individual and social preferences in DCTA acceptance and compliance. Unlike previous work (Jansen-Kosterink, 2020; Zhang et al., 2020), our study focused on individual and social factors and used a representative sample. From a technical standpoint, DCTAs offer direct societal benefits but only indirect individual benefits, because the self-quarantine alerts from the DCTA protect others rather than the user of the DCTA who has been in contact with an infected person. Indirect benefits may exist nevertheless; for instance, people may avoid putting unnecessary strain on their immune system after contact alerts or be prepared for getting sick. Interestingly, the results revealed that acceptance of DCTAs was not related to social preferences, but was rather associated with individual considerations related to risk perceptions and risk preferences. More specifically, our results show the need in this global pandemic to trade off different risks against each other, for example, the risks concerning health and data security. Acceptance of DCTAs was high for individuals perceiving COVID-19 as a severe health threat but low for individuals associating DCTA use with high data-security risks (see also Jansen-Kosterink, 2020; Zhang et al., 2020).

We further found that individuals who were comparably tolerant regarding health risks accepted DCTAs less than those tolerating data-security risks. Contrary to our hypotheses, individuals viewing COVID-19 as a high economic risk were less inclined to accept DCTAs. One reason might be that individuals do not want to know about their own infection risk as this might mean that they have to isolate themselves, possibly having a negative impact on their economic situation. Another reason might be a general societal divide, with people who emphasise the health risks of COVID-19 giving less attention to the economic repercussions and vice versa. Thus, people who are most concerned about the economic risks of COVID-19 might tend to give little support to measures against the COVID-19 pandemic, because they might think they have severe negative economic repercussions. However, this perception appears to be incorrect, as measures to contain the health threat posed by the COVID-19 pandemic ultimately also reduce the long-term economic impact.

In addition to individual and social factors, we found more DCTA acceptance for people who supported the policies to mitigate COVID-19, had a higher affinity for technology, and had a better comprehension of the functionality of the DCTAs. These additional factors had a higher effect on DCTA acceptance than risk perceptions and risk preferences. Our finding that DCTA acceptance increased strongly with policy support is in line with previous results that found trust in the government's datasecurity policies to be a key predictor of DCTA acceptance (Garrett, 2021b; Lewandowsky, 2021). Additionally, it is important to ensure that people comprehend the functionality of DCTAs well, particularly regarding data security, because our results show that the comprehension of DCTAs mildly decreased with higher risk perception in the data-security domain.

Compliance with DCTAs was strong: The results show a high willingness to comply with DCTAs in the sense that upon receiving a warning from the application about a potential risk, people indicated they would follow the suggested measures, such as selfquarantine or testing. Risk-averse individuals and more honest individuals were more likely to comply with DCTAs. However, compliance with the assessment of a DCTA is only fully effective if DCTAs are used, and currently use rates in many European countries are low. One critical factor for taking up a voluntary measure such as a DCTA is acceptance of that measure. As such, while acceptance is above medium in our sample (3.75 on a 1-5 scale), further increasing acceptance of DCTAs must have a high priority.

Recommendations for increasing DCTA acceptance. Our results suggest that the acceptance of DCTAs may be increased by educating people about specific risks and emphasising personal benefits from the use of DCTAs. In doing so, the emphasis should be on the magnitude of health risks to the individual and on the clarification of the comparably low data-security risk posed by DCTAs. Long-term or chronic illness for at least 28 days after a positive COVID-19 test (so-called long COVID) has been shown to affect around 5\% of young people (aged 20-30 years) and has been linked with age: In the age group of 18-49-year-olds, around 10\% suffer from long-term symptoms and this increases to about $22 \%$ for age 70 and above (Sudre, 2021). According to a longitudinal study from the Faroe Islands, around half of COVID-19 patients reported the persistence of at least one symptom 125 days after onset (Petersen, 2020), and according to a cross-sectional study of discharged hospital patients, 
$53 \%$ reported persistent breathlessness, 34\% a cough, and $69 \%$ fatigue after 54 days (Mandal, 2021). A meta-analysis revealed that the progression of COVID-19 is affected by being male, being older than 65 , and smoking in addition to several underlying conditions such as diabetes, cardiovascular disease, and respiratory disease (Zheng, 2020). Individual benefits of using DCTAs, such as protecting people with whom one is close, such as family and friends, or avoiding stress to one's own immune system if one receives a contact alert could also be emphasised. Clarification about data-security risks could compare the DCTAs' data collection, data storage, and data use relative to other frequently-used smartphone apps and social media platforms. Further, efforts to educate the public about how and why DCTAs work and the explanation of the technical details of contact-tracing applications in an accessible way should have a substantial positive effect on their acceptance. In sum, efforts could focus on emphasising individual benefits resulting from DCTAs and educating people so they understand the societal policy in general and the specific measure in particular.

Future empirical research into the uptake of digital contact-tracing devices is needed to examine the effectiveness of potential types of interventions to facilitate DCTA uptake. Regarding interventions that target risk perception, for instance, it is an open question whether effective interventions in the domain of digital contact tracing consist in choice architectures such as nudges (which are effective for climate action, Nisa, 2019), graphical risk and uncertainty communication (Spiegelhalter et al., 2011), social comparisons, or the contextualisation of a novel risk in relation to old risks such as the data security of other mobile applications. Which intervention designs work in the context of digital contact tracing is still an open question.

\section{Data availability}

Additional data and materials are available online (https://osf.io/ u6ngf/).

Received: 17 December 2020; Accepted: 28 June 2021;

Published online: 06 August 2021

\section{Notes}

1 Incorrect answers to several explicit attention-check items or self-reported lack of data quality.

2 Ten percent of the participants were randomly selected together with a counterpart and received the payoff distribution determined by one of their decisions (randomly selected) or the amount assigned to the counterpart.

3 We tested for two moderators. For one, we analysed if the perceived overall effectiveness of the SwissCovid application moderated the main effects on the acceptance of and compliance with DCTAs (as preregistered). Secondly, we analysed if perceiving COVID-19 as a more direct local threat to Switzerland moderated the effects of risk perception on DCTA acceptance and compliance (as preregistered). To this end we used the regression model specification that resulted from the covariate selection and added the respective moderator by including all interaction terms with the moderator variable and the presumably influenced covariates, resulting in two moderator models. For each dependent variable (acceptance and compliance), we compared the fit of the two moderator models to the model without the moderator by the Bayes factor.

\section{References}

Abdelrahman M (2020) Personality traits, risk perception, and protective behaviors of Arab residents of Qatar during the COVID-19 pandemic. Int J Mental Health Addiction. https://doi.org/10.1007/s11469-020-00352-7

Akinbi A, Forshaw M, Blinkhorn V (2020) Contact tracing apps for COVID-19 pandemic: challenges and potential. Preprint at https://doi.org/10.31219/osf.io/6xbcs

Alvarez FE, Argente D, Lippi F (2020) A simple planning problem for COVID-19 lockdown. Working Paper 26981. National Bureau of Economic Research. http://www.nber.org/papers/w26981.

Bavel JJV et al. (2020) Using social and behavioural science to support COVID-19 pandemic response. Nat Hum Behav 4(5):460-471. https://doi.org/10.1038/ s41562-020-0884-Z
Beskorovajnov W et al. (2020) ConTra corona: contact tracing against the cor onavirus by bridging the centralized-decentralized divide for stronger privacy. https://eprint.iacr.org/2020/505.pdf

Betsch C, Wieler LH, Habersaat K (2020) Monitoring behavioural insights related to COVID-19. Lancet 395(10232):1255-1256. https://doi.org/10.1016/S01406736(20)30729-7

Blasimme A, Vayena E (2020) Whatas next for COVID-19 apps? Governance and oversight. Science 370(6518):760-762. https://doi.org/10.1126/science. abd9006

Brooks SK et al. (2020) The psychological impact of quarantine and how to reduce it: rapid review of the evidence. Lancet 395(10227):912-920. https://doi.org/ 10.1016/S0140-6736(20)30460-8

Camerer CF (2003) Behavioural studies of strategic thinking in games. Trends Cogn Sci 7(5):225-231. https://doi.org/10.1016/S1364-6613(03)00094-9

Campos-Mercade P et al. (2021) Prosociality predicts health behaviors during the COVID-19 pandemic. Technical report https://doi.org/10.1016/j. jpubeco.2021.104367

Chen Y, Li SX (2009) Group identity and social preferences. Am Econ Rev 99 (1):431-457. https://doi.org/10.1257/aer.99.1.431

de Vries RE (2013) The 24-item brief HEXACO Inventory (BHI). J Res Pers 47 (6):871-880. https://doi.org/10.1016/j.jrp.2013.09.003

Dryhurst S et al. (2020) Risk perceptions of COVID-19 around the world. J Risk Res 23(7-8):994-1006. https://doi.org/10.1080/13669877.2020.1758193

Falk A, Fischbacher U (2006) A theory of reciprocity. Games Econ Behav 54 (2):293-315. https://doi.org/10.1016/j.geb.2005.03.001

Federal Office of Public Health (FOPH) (2020a) Coronavirus: Information about the Swiss Covid App. https://www.bag.admin.ch/bag/en/home/krankheiten/ ausbrueche-epidemien-pandemien/aktuelle-ausbrueche-epidemien/novelcov/swisscovid-app-und-contact-tracing.html\#-1601404801

Federal Office of Public Health (FOPH) (2020b) Coronavirus: people at especially high risk. https:/www.bag.admin.ch/bag/en/home/krankheiten/ausbruecheepidemien-pandemien/aktuelle-ausbrueche-epidemien/novel-cov/besondersgefaehrdete-menschen.html

Federal Statistical Office (2021) SwissCovid App monitoring. https://www. experimental.bfs.admin.ch/expstat/en/home/innovative-methods/swisscovidapp-monitoring.html

Fehr E, Schmidt KM (1999) A theory of fairness, competition, and cooperation. Q J Econ 114(3):817-868. https://doi.org/10.1162/003355399556151

Ferretti L et al. (2020) Quantifying dynamics of SARS-CoV-2 transmission suggests that epidemic control and avoidance is feasible through instantaneous digital contact tracing. Science 368:eabb6936. https://doi.org/10.1126/science. abb6936

Frey R et al. (2017) Risk preference shares the psychometric structure of major psychological traits. Sci Adv 3(10):e1701381. https://doi.org/10.1126/ sciadv.1701381

Garrett PM et al. (2021a) Young adults view smartphone tracking technologies for COVID-19 as acceptable: the case of Taiwan. Int J Environ Res Public Health 18:3. https://doi.org/10.3390/ijerph18031332

Garrett PM et al. (2021b) The acceptability and uptake of smartphone tracking for COVID-19 in Australia. PLoS ONE 16(1):e0244827. https://doi.org/10.1371/ journal.pone. 0244827

Glöckner A et al. (2020) The perception of infection risks during the early and later outbreak of COVID-19 in Germany: consequences and recommendations. https://doi.org/10.31234/osf.io/wdbgc

Hoffrage U, Gigerenzer G (1998) Using natural frequencies to improve diagnostic inferences. Acad Med 73(5):538-40

Ienca M, Vayena E (2020) On the responsible use of digital data to tackle the COVID-19 pandemic. Nat Med 26(4):463-464. https://doi.org/10.1038/ s41591-020-0832-5

Jansen-Kosterink SM et al. (2020) Predictors to use mobile apps for monitoring COVID-19 symptoms and contact tracing: a survey among Dutch citizens. https://doi.org/10.1101/2020.06.02.20113423v1

Karlsson LC et al. (2021) Fearing the disease or the vaccine: the case of COVID-19. Pers Individ Dif 172:110590. https://doi.org/10.1016/j. paid.2020.110590

Kochańczyk M, Lipniacki T (2021) Pareto-based evaluation of national responses to COVID-19 pandemic shows that saving lives and protecting economy are non-trade-off objectives. Sci Rep 11(1):2425. https://doi.org/10.1038/s41598021-81869-2

Kwok KO et al. (2020) Community responses during early phase of COVID-19 epidemic, Hong Kong. Emerg Infect Dis 26(7):1575. https://doi.org/10.3201/ eid2607.200500

Lewandowsky S et al. (2021) Public acceptance of privacy-encroaching policies to address the COVID-19 pandemic in the United Kingdom. PLoS ONE 16(1): e0245740. https://doi.org/10.1371/journal.pone.0245740

Mandal S et al. (2021) 'Long-COVID': a cross-sectional study of persisting symptoms, biomarker and imaging abnormalities following hospitalisation for COVID-19. Thorax 76(4):396-398 
McFarland S, Webb M, Brown D (2012) All humanity is my ingroup: a measure and studies of identification with all humanity. J Pers Soc Psychol 103(5):830. https://doi.org/10.1037/a0028724

Menni C et al. (2020) Real-time tracking of self-reported symptoms to predict potential COVID-19. Nat Med. 26:1037-1040

Murphy RO, Ackermann KA, Handgraaf M (2011) Measuring social value orientation. Judgm Decis Mak 6(8):771-781

Nisa CF et al. (2019) Meta-analysis of randomised controlled trials testing behavioural interventions to promote household action on climate change. Nat Commun 10(1):4545. https://doi.org/10.1038/s41467-019-12457-2

Ostrom E (1990) Governing the commons: the evolution of institutions for collective action. Cambridge University Press, Cambridge

Park S, Choi GJ, Ko H (2020) Information technology-based tracing strategy in response to COVID-19 in South Korea-privacy controversies. JAMA 323:2129-2130. https://doi.org/10.1001/jama.2020.6602

Pavone F et al. (2020) Using reference models in variable selection. http://arxiv.org/ abs/2004.13118

Petersen MS et al. (2020) Long COVID in the Faroe Islands-a longitudinal study among non-hospitalized patients. Clin Infect Dis. https://doi.org/10.1093/cid/ ciaal 1792

Piironen J, Paasiniemi M, Vehtari A (2020) Projective inference in highdimensional problems: prediction and feature selection. Electron J Stat 14 (1):2155-2197. https://doi.org/10.1214/20-ejs1711

Plohl N, Musil B (2021) Modeling compliance with COVID-19 prevention guidelines: the critical role of trust in science. Psychol Health Med 26 (1):1-12. https://doi.org/10.1080/13548506.2020.1772988

Robert Koch Institut (2021) Kennzahlen zur Corona Warn App. https://www.rki. de/DE/Content/InfAZ/N/Neuartiges_Coronavirus/WarnApp/ Archiv_Kennzahlen/Kennzahlen_26032021.pdf?_blob=publicationFile

Salathé M et al. (2020) COVID-19 epidemic in Switzerland: on the importance of testing, contact tracing and isolation. Swiss Med Wkly 150(11-12):w20225

Spiegelhalter D, Pearson M, Short I (2011) Visualizing uncertainty about the future. Science 333(6048):1393-1400. https://doi.org/10.1126/science.1191181

Sudre CH et al. (2021) Attributes and predictors of long COVID. Nat Med 27:4. https://doi.org/10.1038/s41591-021-01292-y

TNS Infratest Sozialforschung (2009) Leben in Deutschland. Befragung 2009 zur sozialen Lage der Haushalte-2009 Questionnaire.

Van der Pligt J (1996) Risk perception and self-protective behavior. Eur Psychol 1 (1):34-43. https://doi.org/10.1027/1016-9040.1.1.34

van Klingeren F. (2020) Playing nice in the sandbox: on the role of heterogeneity, trust and cooperation in common-pool resources. 15. https://doi.org/10.1371/ journal.pone. 0237870

Vehtari A, Ojanen J (2012) A survey of Bayesian predictive methods for model assessment, selection and comparison. Stat Surv 6:142-228. https://doi.org/ $10.1214 / 12-S S 102$

Visschers VH et al. (2009) Probability information in risk communication: a review of the research literature. Risk Anal 29(2):267-287. https://doi.org/10.1111/ j.1539-6924.2008.01137.x

Weber EU, Milliman RA (1997) Perceived risk attitudes: relating risk perception to risky choice. Manage Sci 43(2):123-144. https://doi.org/10.1287/mnsc.43.2.123

Weinstein ND, Lyon JE (1999) Mindset, optimistic bias about personal risk and health-protective behaviour. Br J Health Psychol 4(4):289-300. https://doi. org/10.1348/135910799168641

Wise T et al. (2020) Changes in risk perception and protective behavior during the first week of the COVID-19 pandemic in the United States. R Soc OpenSci 7:20074. https://doi.org/10.1098/rsos.200742
Xia Y, Lee G (2020) How to return to normalcy: fast and comprehensive contact tracing of COVID-19 through proximity sensing using mobile devices. Preprint at https://arxiv.org/abs/2004.12576

Xie K et al. (2020) The impact of risk perception on social distancing during the COVID-19 pandemic in China. Int J Environ Res Public Health 17(17):6256. https://doi.org/10.3390/ijerph17176256

Zettler I et al. (2021) The role of personality in COVID-19 related perceptions, evaluations, and behaviors: findings across five samples, nine traits, and 17 criteria. https://doi.org/10.1177/19485506211001680

Zhang B, Kreps S, McMurry N (2020) Americans' perceptions of privacy and surveillance in the COVID-19 pandemic. https://doi.org/10.1371/journal. pone. 0242652

Zheng Z et al. (2020) Risk factors of critical \& mortal COVID-19 cases: a systematic literature review and meta-analysis. J Infect 81(2):e16-e25. https:// doi.org/10.1016/j.jinf.2020.04.021

Zhong BL et al. (2020) Knowledge, attitudes, and practices towards COVID-19 among Chinese residents during the rapid rise period of the COVID-19 outbreak: a quick online cross-sectional survey. Int J Biol Sci 16 (10):1745-1752. https://doi.org/10.7150/ijbs.45221

\section{Author contributions}

RA, JBJ, DSM, and JR designed the research and wrote the paper. RA and DSM designed the questionnaire, RA and JBJ conducted the analyses. RA and JBJ contributed equally to the research.

\section{Competing interests}

The authors declare no competing interests.

\section{Additional information}

Supplementary information The online version contains supplementary material available at https://doi.org/10.1057/s41599-021-00856-0.

Correspondence and requests for materials should be addressed to R.A.

Reprints and permission information is available at http://www.nature.com/reprints

Publisher's note Springer Nature remains neutral with regard to jurisdictional claims in published maps and institutional affiliations.

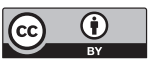

Open Access This article is licensed under a Creative Commons Attribution 4.0 International License, which permits use, sharing, adaptation, distribution and reproduction in any medium or format, as long as you give appropriate credit to the original author(s) and the source, provide a link to the Creative Commons license, and indicate if changes were made. The images or other third party material in this article are included in the article's Creative Commons license, unless indicated otherwise in a credit line to the material. If material is not included in the article's Creative Commons license and your intended use is not permitted by statutory regulation or exceeds the permitted use, you will need to obtain permission directly from the copyright holder. To view a copy of this license, visit http://creativecommons.org/licenses/by/4.0/.

(C) The Author(s) 2021 